\title{
Some Web Intelligence Oriented Brain Informatics Studies
}

\author{
Yulin Qin \\ The International WIC Institute \\ Beijing University of Technology, China, and \\ Department of Psychology, Carnegie Mellon University, USA \\ yulinqin@gmail.com
}

With the advancement both in the Web (e.g., semantic Web and human-level wisdom-Web computing) and in Brain Informatics (BI) (e.g., advanced information technologies for brain science and non-invasive neuroimaging technologies, such as functional magnetic resonance imaging (fMRI)), several lines of BI research have been developed directly or indirectly related to Web Intelligence (WI). Some of them can be treated as the extension to the Web research of the tradition BI research, such as computational cognitive modeling like ACT-R. ACT-R is a theory and model of computational cognitive architecture which consists of functional modules, such as declarative knowledge module, procedural knowledge module, goal module and input (visual, aural), output (motor, verbal) modules. Information can be proposed parallel inside and among the modules, but has to be sequentially if it needs procedural module to coordinate the behavior across modules. At the International WIC Institute (WICI), we are trying to introduce this kind of architecture and the mechanism of activation of the units in declarative knowledge module into our Web information system. Based on or related to ACT-R, theories and models that are with very close relation to WI have also been developed, such as threaded cognition for concurrent multitasking, cognitive agents, human-Web interaction (e.g., SNIT-ACT (Scentbased navigation and information foraging in the ACT cognitive architecture). At the WICI, we are also working on the user behavior and reasoning on the Web by eye-tracker and fMRI. Some of other BI studies, however, have been developed directly by the requirement of WI research. For example, to meet the requirement of the development of Granular Reasoning ( $\mathrm{GrR}$ ) technologies in WI research, people at the WICI have been checking how human can perceive the real world under many levels of granularity (i.e., abstraction) and can also easily switch among granularities. By focusing on different levels of granularity, one can obtain different levels of knowledge, as well as in-depth understanding of the inherent knowledge structure. The interaction between human intelligence inspired WI methodology research and WI stimulated BI principle research will benefit both BI and WI researches greatly. 